\title{
Are discordant positron emission tomography and pathological assessments of the mediastinum in non-small cell lung cancer significant?
}

\author{
Daniel J. Tandberg, BS, ${ }^{\mathrm{a}}$ Nathan G. Gee, MD, ${ }^{\mathrm{b}}$ Junzo P. Chino, MD, ${ }^{\mathrm{a}}$ Thomas A. D'Amico, MD, ${ }^{\mathrm{c}}$ \\ Neal E. Ready, MD, PhD, ${ }^{\mathrm{d}}$ R. Edward Coleman, MD, ${ }^{\mathrm{b}}$ and Chris R. Kelsey, $\mathrm{MD}^{\mathrm{a}}$
}

Objective: Many patients with non-small cell lung cancer have positive mediastinal lymph nodes on preoperative positron emission tomography (PET) but do not have mediastinal involvement after surgery. The prognostic significance of this discordance was assessed.

\begin{abstract}
Methods: This Institutional Review Board-approved study evaluated patients treated with upfront surgery at Duke Cancer Institute (Durham, NC) for non-small cell lung cancer from 1995 to 2008. Those staged with PET with pN0-1 disease after negative invasive mediastinal assessment were included. Mediastinal lymph nodes were scored as positive or negative based on visual analysis of the preoperative PET. Clinical outcomes of the PET-positive and PET-negative cohorts were estimated using the Kaplan-Meier method and compared using a log-rank test. Prognostic factors were assessed using a multivariate analysis.
\end{abstract}

Results: A total of 547 patients were assessed, of whom 105 (19\%) were PET positive in the mediastinum. The median number of mediastinal lymph node stations sampled was 4 (range, 1-9). The 5-year risk of local recurrence was $26 \%$ in PET-positive versus $21 \%$ in PET-negative patients $(P=.50)$. Patterns of local failure were similar between the 2 groups. Distant recurrence $(35 \%$ vs $29 \% ; P=.63)$ and overall survival $(44 \%$ vs $54 \% ; P=.52$ ) were comparable for PET-positive and PET-negative patients. On multivariate analysis, a positive PET was not significant for local recurrence (hazard ratio $[\mathrm{HR}], 1 ; P=1$ ), distant recurrence (HR, $0.82 ; P=.42$ ), or overall survival (HR, 1.08; $P=.62$ ).

Conclusions: Patients with positive mediastinal lymph nodes on preoperative PET, but negative on histologic analysis, are not at increased risk of disease recurrence. Pathologic staging remains the standard. (J Thorac Cardiovasc Surg 2013;146:796-801)

Earn CME credits at

http://cme.ctsnetjournals.org

Fluorodeoxyglucose positron emission tomography (FDGPET) is commonly used in the diagnosis and staging of non-small cell lung cancer (NSCLC). PET has improved preoperative staging of NSCLC in several trials. ${ }^{1-3}$ However, in the assessment of mediastinal lymph nodes, PET has been associated with a significant false-positive rate. Positive predictive values for PET are reported to range from $69 \%$ to $90 \%$, with similar values for integrated PET-computed tomography (CT).$^{4-8}$ Thus, $10 \%$ to $31 \%$ of patients will

From the Department of Radiation Oncology, ${ }^{a}$ the Division of Nuclear Medicine, ${ }^{\mathrm{b}}$ Department of Radiology, the Division of Cardiothoracic Surgery, ${ }^{\mathrm{c}}$ Department of Surgery, and the Division of Medical Oncology, ${ }^{\mathrm{d}}$ Department of Medicine, Duke University School of Medicine, Durham, NC.

Disclosures: R. Edward Coleman (deceased) received research funding and honoraria from General Electric Healthcare. All other authors have nothing to disclose with regard to commercial support.

Received for publication Jan 28, 2013; revisions received May 9, 2013; accepted for publication May 23, 2013; available ahead of print July 18, 2013.

Address for reprints: Daniel J. Tandberg, BS, Department of Radiation Oncology, Duke University School of Medicine, DUMC Box 3085, Durham, NC 27710 (E-mail: d.tandberg@gmail.com).

$0022-5223 / \$ 36.00$

Copyright $(2) 2013$ by The American Association for Thoracic Surgery

http://dx.doi.org/10.1016/j.jtcvs.2013.05.027 have positive mediastinal lymph nodes on preoperative PET but will not have mediastinal disease at surgery.

It is unclear whether positive PET findings are prognostic, despite negative surgical findings. One study showed that patients with positive mediastinal lymph nodes by PET, which were negative at histologic analysis, were at higher risk of disease recurrence. ${ }^{9}$ On the other hand, another study showed that overall and nodal stage, determined by PET, was correlated with survival, but no additional predictive value was obtained when histologic stage was also known. ${ }^{10} \mathrm{We}$ sought to explore this issue further by examining whether patients with positive lymph nodes in the mediastinum by PET, but histologically negative after surgery, are at higher risk for disease recurrence, particularly in the mediastinum.

\section{METHODS}

This Institutional Review Board-approved study evaluated all patients who underwent initial surgery for NSCLC at Duke Cancer Institute (Durham, NC) between 1995 and 2008 (patients receiving preoperative chemotherapy and/or radiotherapy were excluded). Those patients with preoperative PET or PET-CT imaging at our institution with pathologic T1-3 N0-1 disease were identified. All patients had mediastinal lymph nodes assessed at mediastinoscopy and/or surgery. Patients were excluded if they had positive surgical margins or received postoperative radiation therapy because these 2 factors are known to affect the risk of local recurrence. We also excluded patients who died within 30 days of surgery 


\section{Abbreviations and Acronyms \\ FDG = fluorodeoxyglucose \\ HR = hazard ratio \\ NSCLC $=$ non-small cell lung cancer \\ PET = positron emission tomography \\ SUV $=$ standardized uptake value \\ SUVmax $=$ maximum standardized uptake value}

or had a primary lung cancer that was not FDG-avid. Patients who received adjuvant chemotherapy were included.

PET imaging with ${ }^{18} \mathrm{~F}$ FDG was performed using a GE Advance scanner (General Electric Healthcare, Milwaukee, Wis) from 1995 to 2003, and the images were reviewed with a concurrent computed tomographic (CT) scan. From 2003 to 2008, a Discovery ST PET/CT scanner (General Electric Healthcare) was used, and PET images were reviewed with a noncontrast-enhanced CT. Fasting patients with a blood glucose level of less than $200 \mathrm{mg} / \mathrm{dL}$ received an intravenous FDG injection approximately 60 minutes before imaging. The acquisition time was 3 to 4 minutes per bed position, depending on body weight. PET images were reconstructed with corrections for attenuation (CT based or based on rotating germanium-68 source for PET only), scatter, random events, and dead time. Filtered back-projection was used before 1999, and 2 iterations of ordered subset expectation maximization were used subsequently.

All PET studies were interpreted by attending nuclear medicine physicians. Although not standardized, lymph nodes were scored as positive when, by visual analysis, they were associated with FDG uptake higher than mediastinal blood pool levels, with correspondence to a lymph node detected on CT. For this study, patients were classified as PET positive or PET negative in the mediastinum, based on the interpretation recorded in the original PET report. Standardized uptake value (SUV) thresholds were not used to define PET positivity because there is no acceptable cutoff value for determining positive versus negative. The International Association for the Study of Lung Cancer lymph node map was used to delineate the anatomic boundaries of mediastinal lymph node stations for mapping of PET-positive lymph nodes. ${ }^{11}$ Staging was recorded using the 7 th edition of the American Joint Committee on Cancer tumor, node, metastasis (TNM) classification system. ${ }^{12}$

All patients underwent an R0 resection with mediastinal lymph node sampling and/or dissection. Between 1995 and 1997, lung tumor specimens were interpreted by multiple pathologists at Duke University. After 1998, most specimens were interpreted by a single pathologist with a special interest in pulmonary pathology. All patients had routine postsurgical surveillance with imaging, including chest $\mathrm{CT}$, but testing was not standardized. Disease recurrence at the surgical resection margin, ipsilateral hilum, and/or mediastinum was considered a local recurrence. All other sites of recurrence were considered distant metastases. Sites of local recurrence, including specific mediastinal lymph node stations, were identified.

The objective of this study was to determine if patients with positive lymph nodes in the mediastinum by PET, but negative by pathologic analysis, were at increased risk of disease progression. The primary end point of interest was time to local recurrence. Patterns of failure between PET-positive and PET-negative patients were also assessed.

\section{Statistical Analysis}

Study data were collected and managed using REDCap electronic data capture tools hosted at Duke University. ${ }^{13}$ The Fischer exact and Mann-Whitney $U$ tests were used to compare patient and treatment characteristics between PET-positive and PET-negative cohorts. The Kaplan-Meier product-limit method was used to estimate 5-year recurrence probabilities and confidence intervals. ${ }^{14}$ Time to local failure and time to distant failure were calculated from the date of surgery to the date of local or distant recurrence, respectively. Local and distant recurrences were scored independently (ie, patients developing a distant recurrence were not censored for local failure, but were assessed for local failure until the date of last follow-up or death).

A Cox multivariate analysis was also performed to assess for clinical and pathologic factors associated with an increased risk of local failure after resection, including mediastinal PET status. Variables were included in the multivariate model if they achieved a $P \leq .15$ on univariate analysis. All statistical tests were 2 sided, and $P<.05$ was considered statistically significant. SAS 9.1 statistical software (SAS Institute, Inc, Cary, NC) was used for statistical analyses.

\section{RESULTS}

There were 547 patients who met the inclusion criteria. The median follow-up was 29 months (range, 1-170 months). Patients were pN0 in $469(86 \%)$ and pN1 in 78 $(14 \%)$. Adjuvant chemotherapy was administered to 57 $(10 \%)$. Other patient characteristics and surgical/pathologic details are shown in Table 1.

Preoperative imaging consisted of PET with concurrent CT $(n=269)$ or integrated PET-CT $(n=278)$. The preoperative PET was positive in the mediastinum in $105(19 \%)$ of the patients and negative in $442(81 \%)$ of the patients. Among PET-positive patients, the median number of mediastinal stations with hypermetabolic lymph nodes was 1 (range, 1-7). The most common mediastinal lymph node stations involved were $4 R(n=58), 5(n=32)$, and $7(n=26)$. The number of patients with positive lymph nodes in other mediastinal stations was as follows: $2 R(n=9), 2 L(n=4), 3(n=8)$, $4 \mathrm{~L}(\mathrm{n}=15), 6(\mathrm{n}=3)$, and $8(\mathrm{n}=4)$.

Mediastinal assessment included mediastinoscopy alone $(\mathrm{n}=83)$, video-assisted thoracoscopic surgery/thoracotomy alone $(n=99)$, or both $(n=365)$. The median number of mediastinal lymph node stations examined was 4 (range, 1-8) in PET-positive patients and 3 (range, 1-9) in PET-negative patients $(P=.042)$. At least 3 mediastinal lymph node stations were sampled in $88 \%$ of PET-positive patients and $78 \%$ of PET-negative patients. Of lymph node stations positive on preoperative PET imaging, 70\% (113/161) were sampled at mediastinoscopy and/or surgery. The most common lymph node station hypermetabolic on PET, but not sampled during the preoperative work-up and/or at surgery, was level $5(21 / 48)$. In $81 \%(17 / 21)$ of cases, when level 5 was not sampled, the patient had a right-sided primary tumor.

Local disease recurrence was identified in 78 patients. The 5-year risk of local recurrence in all patients was $22 \%$ (95\% confidence interval $[\mathrm{CI}], 17 \%-27 \%$ ). There was no significant difference in risk of local recurrence between the PET-positive patients and PET-negative patients $(26 \%$ vs $21 \% ; P=.50$ ) (Figure 1). This was true in both the pN0 subgroup $(P=.63)$ and the $\mathrm{pN1}$ subgroup $(P=.97)$.

Patterns of local recurrence were similar between PET-positive and PET-negative patients (Table 2). Among the 105 PET-positive patients, 14 had a local disease recurrence in the mediastinum. Imaging studies could be 
TABLE 1. Patient and treatment characteristics

\begin{tabular}{|c|c|c|c|c|}
\hline \multirow[b]{2}{*}{ Parameter } & \multirow[b]{2}{*}{ All $(n=547)$} & \multicolumn{2}{|c|}{ PET mediastinum findings } & \multirow[b]{2}{*}{$P$ value } \\
\hline & & Positive $(n=105)$ & Negative $(n=442)$ & \\
\hline Sex & & & & .3858 \\
\hline Male & $280(51)$ & $58(55)$ & $222(50)$ & \\
\hline Female & $267(49)$ & $47(45)$ & $220(50)$ & \\
\hline Age, median (range), y & $68(39-93)$ & $69(40-86)$ & $68(39-93)$ & 6457 \\
\hline PET imaging technique & & & & .5885 \\
\hline PET & $269(49)$ & $49(47)$ & $220(50)$ & \\
\hline PET-CT & $278(51)$ & $56(53)$ & $222(50)$ & \\
\hline Mediastinoscopy & $448(82)$ & $93(89)$ & $355(80)$ & .0491 \\
\hline Surgical procedure & & & & .1192 \\
\hline Wedge/segmentectomy & $94(17)$ & $20(19)$ & $74(17)$ & \\
\hline Lobectomy* & $428(78)$ & $76(72)$ & $352(80)$ & \\
\hline Pneumonectomy & $25(5)$ & $9(9)$ & $16(3)$ & \\
\hline Surgical approach & & & & .0456 \\
\hline Open & $217(40)$ & $51(49)$ & $166(38)$ & \\
\hline VATS & $330(60)$ & $54(51)$ & $276(62)$ & \\
\hline $\begin{array}{l}\text { No. of mediastinal LN stations } \\
\text { sampled, median (range) }\end{array}$ & $4(1-9)$ & $4(1-8)$ & $3(1-9)$ & .0416 \\
\hline Histology & & & & .1372 \\
\hline Adenocarcinoma & $264(49)$ & $42(40)$ & $222(51)$ & \\
\hline Squamous cell carcinoma & $208(38)$ & $47(45)$ & $161(36)$ & \\
\hline Large-cell carcinoma & $38(7)$ & $11(11)$ & $27(6)$ & \\
\hline NSCLC NOS & $30(6)$ & $4(4)$ & $26(6)$ & \\
\hline Histologic differentiation & & & & .0873 \\
\hline Well & $46(9)$ & $4(4)$ & $42(10)$ & \\
\hline Moderate & $245(45)$ & $43(41)$ & $202(46)$ & \\
\hline Poor & $187(34)$ & $45(43)$ & $142(32)$ & \\
\hline Lymphovascular space invasion & $111(20)$ & $27(26)$ & $84(19)$ & .1377 \\
\hline Visceral pleural involvement & $126(23)$ & $22(21)$ & $104(24)$ & .6086 \\
\hline Pathologic nodal stage & & & & .5381 \\
\hline No & $469(86)$ & $88(84)$ & $381(86)$ & \\
\hline N1 & $78(14)$ & $17(16)$ & $61(14)$ & \\
\hline Pathologic stage & & & & .0006 \\
\hline IA & $242(44)$ & $38(36)$ & $204(46)$ & \\
\hline IB & $146(27)$ & $23(22)$ & $123(28)$ & \\
\hline IIA & $98(18)$ & $20(19)$ & $78(18)$ & \\
\hline IIB & $49(9)$ & $20(19)$ & $29(6)$ & \\
\hline IIIA & $12(2)$ & $4(4)$ & $8(2)$ & \\
\hline Postoperative chemotherapy & $57(10)$ & $17(16)$ & $40(9)$ & .0486 \\
\hline
\end{tabular}

Values are given as number (\%) unless otherwise indicated. PET, Positron emission tomography; CT, computed tomography; VATS, video-assisted thoracoscopic surgery; $L N$, lymph node; NSCLC, non-small cell lung cancer; NOS, not otherwise specified. *Lobectomy, bilobectomy, or sleeve lobectomy.

retrieved in 13 of these patients to determine the specific site(s) of mediastinal failure. In 11 of the 13 patients, the site of mediastinal failure correlated to the lymph node station positive on preoperative PET. Most (10/11) of these patients had undergone surgical sampling of that mediastinal lymph node station at surgery.

For all patients, the 5-year risk of developing distant metastases was $30 \%(95 \% \mathrm{CI}, 25 \%-35 \%)$ and the 5-year overall survival was $52 \%$ (95\% CI, $47 \%-57 \%)$. No differences in risk of distant recurrence ( $35 \%$ vs $29 \% ; P=.63$ ) or overall survival $(44 \%$ vs $54 \% ; P=.52)$ (Figure 2 ) were noted between the PET-positive and PET-negative groups (Figure 2).
On multivariate analysis, PET positivity in the mediastinum was not associated with increased risk of local recurrence (hazard ratio [HR], 1.00; $P=.99$ ), distant recurrence (HR, $0.82 ; P=.42$ ), or overall survival (HR, 1.08; $P=.62$ ) (Table 3).

For the subset of patients $(n=453)$ undergoing at least a lobectomy (excluding wedge or segmentectomy), there continued to be no difference in 5-year risk of local disease recurrence, distant recurrence, or overall survival between the PET-positive and PET-negative cohorts. On multivariate analysis, PET positive in the mediastinum was not associated with increased risk of local recurrence in this subgroup (HR, $0.78 ; 95 \% \mathrm{CI}, 0.41-1.48 ; P=.45$ ). 


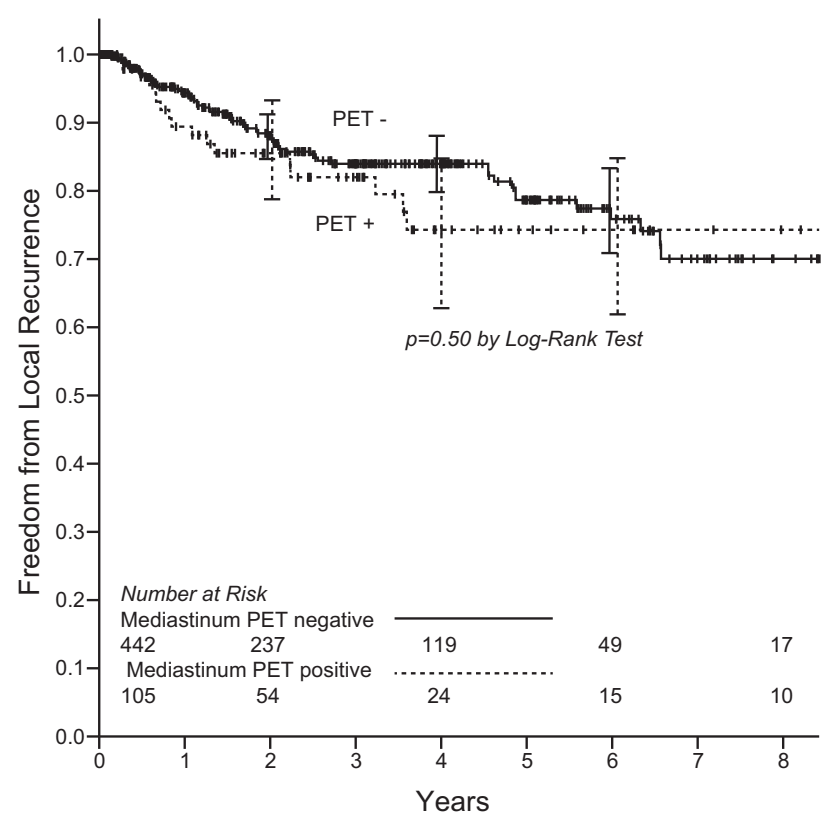

FIGURE 1. Kaplan-Meier curves with $95 \%$ confidence intervals comparing freedom from local recurrence for patients with pathologic N0-N1 non-small cell lung cancer with either positron emission tomographic (PET)-positive (dotted line) or PET-negative (solid line) mediastinal lymph nodes on preoperative PET.

Current guidelines recommend systematic sampling of at least 3 lymph node stations in early-stage NSCLC. ${ }^{15,16}$ In the subset of patients $(n=439)$ who had 3 or more lymph node stations sampled, there was no difference in 5-year rates of local recurrence $(23 \%$ vs $22 \% ; P=.94)$, distant recurrence $(34 \%$ vs $30 \% ; P=.88)$, and overall survival $(48 \%$ vs $52 \% ; P=.44)$ for PET-positive versus PET-negative patients, respectively. Also, contralateral mediastinal disease is rare in patients with early-stage, right-sided tumors. ${ }^{17}$ After excluding the patients with right-sided tumors and contralateral PET-positive mediastinal lymph nodes $(n=22)$, there continued to be no difference in clinical outcomes.

\section{DISCUSSION}

In this analysis, we observed that $19 \%$ of patients with NSCLC were apparently overstaged in the mediastinum

TABLE 2. Patterns of local failure

\begin{tabular}{lccc}
\hline & All & \multicolumn{2}{c}{ PET mediastinum findings } \\
\cline { 3 - 4 } \multicolumn{1}{c}{ Parameter* $^{*}$} & $\begin{array}{c}\text { Positive } \\
(\mathbf{n}=\mathbf{5 4 7})\end{array}$ & $\begin{array}{c}\text { Negative } \\
(\mathbf{n}=\mathbf{4 4 2})\end{array}$ \\
\hline Local recurrence (all sites) & $78(14)$ & $17(16)$ & $61(14)$ \\
Mediastinum & $54(69)$ & $14(82)$ & $40(66)$ \\
Ipsilateral hilum & $24(31)$ & $7(41)$ & $17(28)$ \\
Stump/surgical line & $24(31)$ & $4(24)$ & $20(33)$ \\
\hline
\end{tabular}

PET, Positron emission tomography. *Values are given as number (\%). Total percentages add up to more than $100 \%$ because some patients had multiple sites of failure.

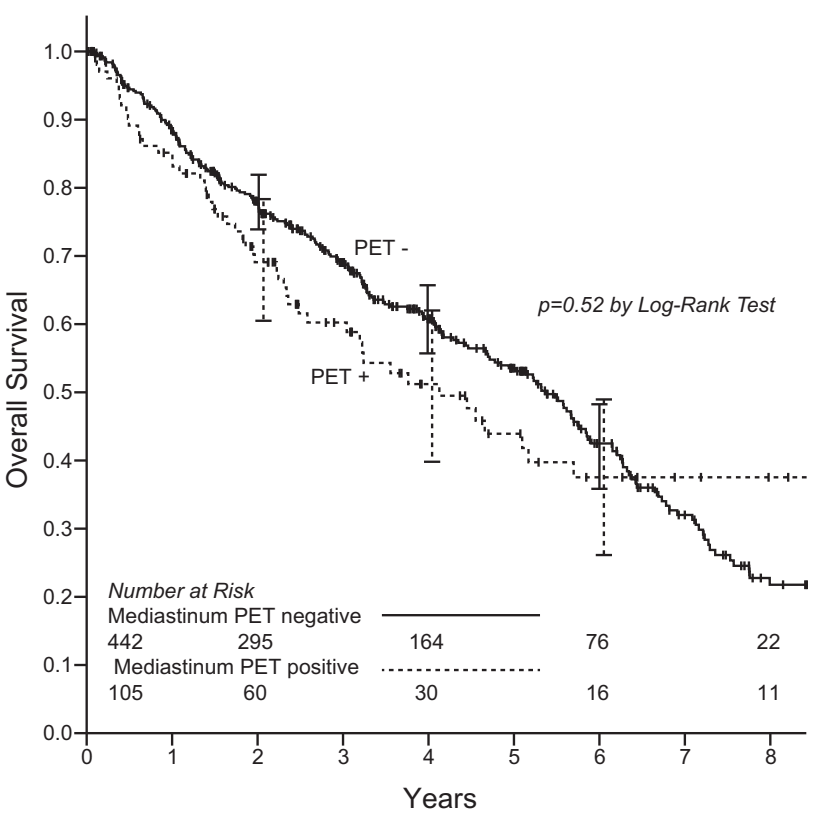

FIGURE 2. Kaplan-Meier curves with $95 \%$ confidence intervals comparing overall survival for patients with pathologic N0-N1 non-small cell lung cancer with either positron emission tomographic (PET)-positive (dotted line) or PET-negative (solid line) mediastinal lymph nodes on preoperative PET.

by PET. Because surgery is the gold standard, it is assumed that this represents false-positive findings (ie, benign lymph nodes with FDG avidity). It is also feasible that the PET accurately identified malignant lymph nodes, but either the involved lymph node was not removed at surgery or the pathologic assessment was inaccurate. However, we found that patients with positive mediastinal findings on preoperative PET, but with pathologic N0-N1 disease, are at no higher risk of disease recurrence or death compared with patients with a negative preoperative PET. Patterns of local recurrence were also not different between the 2 cohorts.

The false-positive rate of PET in this study is similar to reports elsewhere in the literature. In large reviews, the false-positive rate for PET in the mediastinum is $10 \%$ to $31 \%{ }^{4-6}$ An integrated PET-CT has been more accurate than PET in determining nodal status in both patient and nodal analyses. ${ }^{18}$ However, such as PET alone, PET-CT is associated with false-positive findings in the mediastinum. ${ }^{7,8}$ There are multiple possible explanations for false-positive PET findings. These include nonmalignant lung diseases, such as tuberculosis, bacterial and fungal lung infections, and sarcoidosis. ${ }^{19,20}$ In a previous study performed at Duke, pneumonia, granulomatous disease, and silicosis were specifically associated with falsepositive PET findings. ${ }^{21}$ Furthermore, PET interpretation is subject to significant interobserver and intraobserver variations. $^{22}$ Some of the variation may be explained by 
TABLE 3. Univariate and multivariate analyses for local recurrence

\begin{tabular}{|c|c|c|c|c|c|c|}
\hline \multirow[b]{2}{*}{ Factors* } & \multicolumn{3}{|c|}{ Univariate analysis } & \multicolumn{3}{|c|}{ Multivariate analysis } \\
\hline & Hazards ratio & $\mathbf{9 5} \% \mathrm{CI}$ & $P$ value & Hazards ratio & $\mathbf{9 5} \% \mathrm{CI}$ & $P$ value \\
\hline Age & 0.99 & $0.96-1.01$ & .2767 & & & \\
\hline \multicolumn{7}{|l|}{ Sex } \\
\hline $\begin{array}{l}\text { Female } \\
\text { Male }\end{array}$ & 1.16 & $0.74-1.81$ & .5209 & & & \\
\hline PET-positive mediastinum & 1.20 & $0.70-2.06$ & .5029 & 0.96 & $0.55-1.69$ & .8993 \\
\hline \multicolumn{7}{|l|}{ Surgical procedure } \\
\hline $\begin{array}{l}\geq \text { Lobectomy } \\
\text { Wedge/segmentectomy }\end{array}$ & 1.47 & $0.84-2.58$ & .1816 & 2.68 & $1.42-5.05$ & .0022 \\
\hline \multicolumn{7}{|l|}{ Surgical approach } \\
\hline $\begin{array}{l}\text { Open } \\
\text { VATS }\end{array}$ & 0.81 & $0.52-1.26$ & .3517 & & & \\
\hline No. of mediastinal LNs sampled & 0.96 & $0.83-1.11$ & 6099 & & & \\
\hline Stage overall $\dagger$ & - & - & $<.0001$ & - & - & $<.0001$ \\
\hline \multicolumn{7}{|l|}{ Histology } \\
\hline $\begin{array}{l}\text { Nonsquamous } \\
\text { Squamous or large cell }\end{array}$ & 2.03 & $1.30-3.19$ & .0020 & 1.78 & $1.13-2.82$ & .0133 \\
\hline Lymphovascular invasion & 1.01 & $0.58-1.75$ & .9785 & & & \\
\hline Visceral pleural invasion & 1.96 & $1.22-3.15$ & .0057 & 1.44 & $0.83-2.48$ & .1920 \\
\hline Adjuvant chemotherapy & 1.66 & $0.91-3.01$ & .0958 & 1.10 & $0.57-2.10$ & .7783 \\
\hline
\end{tabular}

CI, Confidence interval; VATS, video-assisted thoracoscopic surgery; $L N$, lymph node; PET, positron emission tomography. $*$ The first factor level listed is the reference set (when multiple factors are listed). †The multivariate analysis hazards ratios for stages are as follows: IB vs IA, 1.51; IIA vs IA, 4.60; IIB vs IA, 4.24; and IIIA vs IA, 1.30.

the lack of consensus for defining a positive lymph node and variation in scanners and their accuracy/resolution. ${ }^{19}$

Our study was limited to patients who underwent invasive staging of the mediastinum during mediastinoscopy and/or surgery. The median number of mediastinal lymph node stations sampled was 4 , and $80 \%$ of patients had 3 or more mediastinal lymph nodes sampled. Patients with PET-positive mediastinal lymph nodes had more extensive evaluation of the mediastinum, suggesting awareness of the surgeon of the PET-positive mediastinal lymph nodes or differences in other prognostic factors (eg, $\mathrm{T}$ stage). Evidence-based guidelines recommend pathologic evaluation of a minimum of 3 mediastinal lymph node stations. ${ }^{15,16}$ To control for variations in mediastinal assessment, we performed a subset analysis including patients who had 3 or more lymph node stations sampled, thus eliminating all patients who were not adequately surgically staged in the mediastinum (patients more likely to have false-negative invasive staging). In this analysis, we continued to see no significant difference in outcomes between PET-negative and PET-positive patients. These data suggest that PET does not provide additional prognostic data beyond the pathologic stage when patients are adequately staged at surgery.

Guidelines also recommend that discrete mediastinal lymph nodes positive on preoperative PET should be sampled pathologically. ${ }^{16,23}$ Although $88 \%$ of PETpositive patients had 3 or more mediastinal lymph node stations sampled invasively in our series, $30 \%$ of discrete mediastinal lymph node stations positive on preoperative
PET were not sampled pathologically. There are several possible explanations for this discrepancy: (1) no lymph nodes identified during invasive assessment at a suspicious mediastinal station, (2) an unusual lymph node pattern that is discounted (PET-positive findings in level 5, with right-sided tumors), (3) confusion regarding which lymph node stations were deemed suspicious, and (4) errors in recording the site of a positive lymph node (radiology and/or pathology report). Furthermore, sampling the hypermetabolic lymph node within a station is vital, but often difficult to confirm, especially retrospectively.

Limited research has assessed the prognostic significance of discordant nodal stages determined by PET and pathologic assessment. One retrospective study showed that both tumor and nodal stages, determined by visual PET interpretation, were correlated with survival. ${ }^{10}$ However, when histologic stage was included in a multivariate model, PET provided no additional prognostic information.

A more recent study by Xie and colleagues ${ }^{9}$ specifically focused on patients with pathologic N0/N1 NSCLC. They demonstrated increased rates of local-regional and distant failures in patients with positive mediastinal findings on preoperative PET, compared with those with a negative PET. In their multivariate analysis, PET positivity in the mediastinum was significant for distant failure and was marginally significant for local-regional failure (HR, 1.9; $P=.093$ ). In our analysis, we did not see significant differences in local recurrence, distant recurrence, or overall survival between the PET-positive and PET-negative cohorts. The most plausible explanation for the discordant results presented by Xie 
and colleagues ${ }^{9}$ and this analysis is sample size. Of 90 patients in their study, only 13 were PET positive in the mediastinum. Thus, their findings were based on a few events. In contrast, we studied 547 patients, of which 105 were PET positive in the mediastinum. It is also possible that differences in the distribution of stage and PET findings contributed to the discordant results. Xie and colleagues ${ }^{9}$ had more patients with $\mathrm{pN} 1$ disease, and these patients were more likely to have a positive PET than patients who were $\mathrm{pNO}$. In contrast, in our study, patients with $\mathrm{pN} 0$ and $\mathrm{pN} 1$ disease had a similar likelihood of having a positive PET. Other possible reasons for discordant results could include differences in PET interpretation, thoroughness of mediastinal sampling/dissection, and/or extent of pathologic assessment.

Our study has several clinical implications. Guidelines recommend invasive assessment of mediastinal lymph node stations positive on staging PET. With a $19 \%$ false-positive rate, our study supports this guideline. Furthermore, our results suggest that histologic findings should be the primary means of assessing prognosis after surgery and guiding adjuvant treatment decisions, even when PET findings appear to conflict with the final pathologic assessment. Thus, PET positivity by itself should not be used as a criterion for adjuvant chemotherapy or radiation therapy when mediastinal lymph nodes are pathologically negative.

There are inherent limitations to this study based on its retrospective design. We used the original PET interpretation to score patients as having PET-positive mediastinal lymph nodes. This would have the most clinical relevance because the original interpretation was the tool used by clinicians making treatment decisions. We did not use SUVmax data in this analysis because no clear cutoff level has been established for determining positive versus negative. In the study by Xie and colleagues, ${ }^{9}$ SUVmax of the mediastinal lymph nodes was not prognostic for local/regional or distant failure. To the best of our knowledge, this study represents the largest study of its kind evaluating discordant PET and histologic results in NSCLC.

\section{CONCLUSIONS}

Positive mediastinal lymph nodes on preoperative PET do not provide any additional prognostic information in patients found to be pathologically negative after curative surgery. Thus, pathologic staging should remain the standard when making adjuvant treatment recommendations.

\section{References}

1. Vesselle H, Pugsley JM, Vallieres E, Wood DE. The impact of fluorodeoxyglucose $\mathrm{F} 18$ positron-emission tomography on the surgical staging of non-small cell lung cancer. $J$ Thorac Cardiovasc Surg. 2002;124:511-9.

2. van Tinteren H, Hoekstra OS, Smit EF, van den Bergh JH, Schreurs AJ, Stallaert RA, et al. Effectiveness of positron emission tomography in the preoperative assessment of patients with suspected non-small-cell lung cancer: the PLUS multicentre randomised trial. Lancet. 2002;359:1388-93.

3. Fischer B, Lassen U, Mortensen J, Larsen S, Loft A, Bertlesen A, et al. Preoperative staging of lung cancer with combined PET-CT. $N$ Engl J Med. 2009;361: $32-9$.

4. Toloza EM, Harpole L, McCrory DC. Noninvasive staging of non-small cell lung cancer: a review of the current evidence. Chest. 2003;123:137S-46S.

5. Fischer BM, Mortensen J, Hojgaard L. Positron emission tomography in the diagnosis and staging of lung cancer: a systematic, quantitative review. Lancet Oncol. 2001;2:659-66.

6. Dwamena BA, Sonnad SS, Angobaldo JO, Wahl RL. Metastases from non-small cell lung cancer: mediastinal staging in the 1990s-meta-analytic comparison of PET and CT. Radiology. 1999;213:530-6.

7. Lv YL, Yuan DM, Wang K, Miao XH, Qian Q, Wei SZ, et al. Diagnostic performance of integrated positron emission tomography/computed tomography for mediastinal lymph node staging in non-small cell lung cancer: a bivariate systematic review and meta-analysis. J Thorac Oncol. 2011;6:1350-8.

8. De Wever W, Stroobants S, Coolen J, Verschakelen JA. Integrated PET/CT in the staging of nonsmall cell lung cancer: technical aspects and clinical integration. Eur Respir J. 2009;33:201-12.

9. Xie LY, Saynak M, Veeramachaneni NK, Fried DV, Jaqtap MR, Chiu WK, et al Non-small cell lung cancer: prognostic importance of positive FDG PET findings in the mediastinum for patients with N0-N1 disease at pathologic analysis. Radiology. 2011;261:226-34.

10. Dunagan DP, Chin R, McCain TW, Case L, Harkness B, Oaks T, et al. Staging by positron emission tomography predicts survival in patients with non-small cell lung cancer. Chest. 2001;119:333-9.

11. Rusch VW, Asamura H, Watanabe H, Giroux DJ, Rami-Porta R, Goldstraw P, et al. The IASLC lung cancer staging project: a proposal for a new international lymph node map in the forthcoming seventh edition of the TNM classification for lung cancer. J Thorac Oncol. 2009;4:568-77.

12. Edge SB, Byrd DR, Compton CC, Fritz AG, Greene FL, Trotti A, eds. AJCC Cancer Staging Manual. 7th ed. New York, NY: Springer; 2010.

13. Harris PA, Taylor R, Thielke R, Payne J, Gonzalez N, Conde JG. Research electronic data capture (REDCap): a metadata-driven methodology and workflow process for providing translational research informatics support. J Biomed Inform. 2009;42:377-81.

14. Collett D. Modeling Survival Data in Medical Research. 2nd ed London, England: Chapman \& Hall; 2003.

15. Whitson BA, Groth SS, Maddaus MA. Surgical assessment and intraoperative management of mediastinal lymph nodes in non-small cell lung cancer. Ann Thorac Surg. 2007;84:1059-65.

16. Ettinger DS, Akerley W, Bepler G, Blum MG, Chang A, Cheney RT, et al. Non small cell lung cancer. J Natl Compr Canc Netw. 2010;8:740.

17. Kelsey CR, Light KL, Marks LB. Patterns of failure after resection of non-smallcell lung cancer: implications for postoperative radiation therapy volumes. Int J Radiat Oncol Biol Phys. 2006;65:1097-105.

18. Cerfolio RJ, Ojha B, Bryant AS, Raghuveer V, Mountz JM, Bartolucci AA. The accuracy of integrated PET-CT compared with dedicated PET alone for the staging of patients with nonsmall cell lung cancer. Ann Thorac Surg. 2004;78: 1017-23.

19. Roberts PF, Follette DM, von Haag D, Park JA, Valk PE, Pounds TR, et al. Factors associated with false-positive staging of lung cancer by positron emission tomography. Ann Thorac Surg. 2000;70:1154-9; discussion 1159-60.

20. Gonzalez-Stawinski GV, Lemaire A, Merchant F, O'Halloran E, Coleman RE Harpole DH, et al. A comparative analysis of positron emission tomography and mediastinoscopy in staging non-small cell lung cancer. $J$ Thorac Cardiovasc Surg. 2003;126:1900-5.

21. Gonzalez-Stawinski GV, Lemaire A, Merchant F, O'Halloran E, Coleman RE, Harpole DH, et al. A comparative analysis of positron emission tomography and mediastinoscopy in staging non-small cell lung cancer. J Thorac Cardiovasc Surg. 2003;126:1900-4.

22. Smulders SA, Gundy CM, van Lingen A, Comans EF, Smeenk FW, Hoekstra OS, et al. Observer variation of 2-deoxy-2-F-18 fluoro-D-glucose-positron emission tomography in mediastinal staging of non-small cell lung cancer as a function of experience, and its potential clinical impact. Mol Imaging Biol. 2007;9:318-22.

23. Detterbeck FC, Jantz MA, Wallace M, Vansteenkiste J, Silvestri GA. Invasive mediastinal staging of lung cancer: ACCP evidence-based clinical practice guidelines (2nd edition). Chest. 2007;132:202S-20S. 